\title{
Effect of microstructural evolution on magnetic properties of Ni thin films
}

\author{
PRASHANT KUMAR, M GHANASHYAM KRISHNA* and A K BHATTACHARYA ${ }^{\dagger}$ \\ School of Physics, University of Hyderabad, Hyderabad 500 046, India \\ ${ }^{\dagger}$ Department of Engineering Sciences, Oxford University, Parks Road, Oxford, UK
}

\begin{abstract}
The magnetic properties of $\mathrm{Ni}$ thin films, in the range $20-500 \mathrm{~nm}$, at the crystalline-nanocrystalline interface are reported. The effect of thickness, substrate and substrate temperature has been studied. For the films deposited at ambient temperatures on borosilicate glass substrates, the crystallite size, coercive field and magnetization energy density first increase and achieve a maximum at a critical value of thickness and decrease thereafter. At a thickness of $50 \mathrm{~nm}$, the films deposited at ambient temperature onto borosilicate glass, MgO and silicon do not exhibit long-range order but are magnetic as is evident from the non-zero coercive field and magnetization energy. Phase contrast microscopy revealed that the grain sizes increase from a value of $30-50 \mathrm{~nm}$ at ambient temperature to $120-150 \mathrm{~nm}$ at $503 \mathrm{~K}$ and remain approximately constant in this range up to $593 \mathrm{~K}$. The existence of grain boundary walls of width $30-50 \mathrm{~nm}$ is demonstrated using phase contrast images. The grain boundary area also stagnates at higher substrate temperature. There is pronounced shape anisotropy as evidenced by the increased aspect ratio of the grains as a function of substrate temperature. Nickel thin films of $50 \mathrm{~nm}$ show the absence of long-range crystalline order at ambient temperature growth conditions and a preferred [111] orientation at higher substrate temperatures. Thin films are found to be thermally relaxed at elevated deposition temperature and having large compressive strain at ambient temperature. This transition from nanocrystalline to crystalline order causes a peak in the coercive field in the region of transition as a function of thickness and substrate temperature. The saturation magnetization on the other hand increases with increase in substrate temperature.
\end{abstract}

Keywords. Magnetic thin films; thermal evaporation; substrate temperature; atomic force microscopy; phase contrast imaging.

\section{Introduction}

Elemental nickel and nickel based thin films are extremely important for a variety of magnetic applications (Chang et al 2003; Ravinder et al 2003; Guan et al 2005; Amoruso et al 2005; Hsieh et al 2005; Sedlácková et al 2005; Girgis et al 2006; Flynn and Desmulliez 2006; Shalyguina et al 2006; Gupta et al 2007; Zhang et al 2007). The scaling down of thin film based devices requires the deposition of these films to lower and lower thicknesses, thereby throwing up new challenges in terms of the evolution of microstructure and structure and the properties of interest, both electrical and magnetic. It is known that the early stages of thin film formation, i.e. in the thickness range of 5-50 $\mathrm{nm}$, is very sensitive to the processing techniques and parameters used (Dirks and Leamy 1977; Messier et al 1984; Snyder et al 1991; Hong et al 1997; Novikov 1999; Seel et al 2000; Klapetek et al 2007; Yang et al 2007). The microstructural and structural parameters that influence the performance of magnetic devices involving thin $(5-50 \mathrm{~nm})$ films include surface

\footnotetext{
*Author for correspondence (mgksp@uohyd.ernet.in)
}

roughness, crystallographic texture, morphology, shape anisotropy, grain size and shapes. The influence of substrate temperature on the growth and magnetic properties of thin films has been studied intensively and it has also been established that properties such as coercivity, remanence, and saturation magnetization are strongly dependent on the microstructure, crystallographic texture and the processing parameters under which the films are deposited (Shimizu et al 1999; Haque et al 2001; Zhao et al 2001; Swerts et al 2002; Wang et al 2002; Lukaszew et al 2003; Serrona et al 2003; Castaldi et al 2004; Yi et al 2004; Aurongzeb et al 2005; Chan et al 2006; Ha et al 2006). There are, however, some issues for which experimental evidence is still lacking. For example, there is very little evidence for the grain and grain boundary evolution in the early stages of $\mathrm{Ni}$ film formation. The critical thickness for crystallization of $\mathrm{Ni}$ is also not very clear. The role of the film-substrate interface in determining the magnetic properties of the films is also not well understood.

The objective of the present work therefore is to establish the nature of grain growth at thickness of the order of $50 \mathrm{~nm}$, the existence (or the lack) of a critical thickness for crystallization and the strain and roughness pro- 
file for Ni films grown by evaporation onto borosilicate glass (BSG), Si (111) and MgO (001) substrates at ambient and elevated temperatures. A correlation with magnetic behaviour of the films is also made. Phase contrast microscopy is demonstrated as a viable technique for monitoring the grain boundary evolution in very thin films.

\section{Experimental}

Nickel thin films of thickness range 20-500 nm have been coated onto different substrates viz. borosilicate glass, silicon, and $\mathrm{MgO}$. To look into substrate temperature effect, nickel thin films of thickness, $50 \mathrm{~nm}$, were coated onto borosilicate glass substrates at temperatures varying from ambient to $593 \mathrm{~K}$. A resistive thermal evaporation unit (Cooke's vacuum products, CV $600 \mathrm{M}$ ) was used for coating at deposition rates ranging from $0 \cdot 1 \AA / \mathrm{s}$ to $0.4 \AA / \mathrm{s}$ and even bumps were controlled up to $3 \AA / \mathrm{s}$. Base pressures were kept in the range 1 to $2 \times$ $10^{-6}$ Torr. The films were evaporated from a tungsten spiral boat onto substrates at a distance of $13 \mathrm{~cm}$. This distance is quite large to yield very small rate of deposition and hence good control. Thickness of films were measured by quartz crystal thickness monitor (Hindhivac thickness monitor model DTM-101) and confirmed in a profilometer after deposition (Ambios technology, XP-1) with a resolution of $\pm 1 \mathrm{~nm}$.

X-ray diffractometry was done with powder X-ray diffractometer (INEL, XRG 3000), with cobalt $K_{\alpha}$ line. Debye-Scherrer's formula was used to calculate the average crystallite size: $a=(k \lambda) /(\beta \cos \theta)$, where $k=0.94$, $\lambda=1.78 \AA, \beta$ is the full width at half maximum in radian and $\theta$ the angle of incidence. A silicon standard was used for calibration to take into account instrumental broadening. The residual strain was calculated from

$$
\varepsilon=(\Delta d) / d=\left(d-d_{\text {bulk }}\right) / d_{\text {bulk }},
$$

where $d$ is the interplanar spacing.

Quantitative information about magnetization was achieved from vibrating sample magnetometer (Lakeshore Cryotronics, Model no. 7400) having sensitivity in the range of $\mu \mathrm{emu}$. Air-gap between magnets is fixed at $3 \mathrm{~cm}$ and for low magnetic moment samples ( $\mu \mathrm{emu}$ ), high time constants that permit lower field sweeping rates were used. The changes in flux caused by the vibrations are translated into a change in current in pick-up coils of the instruments, which registers a particular magnetization. To achieve large signal to noise ratio, we have taken large area of the sample covering total area of sample holder. A field limit of $\pm 0.5 \mathrm{~T}$ in the steps of 0.005 or $0.01 \mathrm{~T}$ was adopted to achieve better accuracy. Hysteresis plots were taken in parallel orientation of films. Volume of the thin film has been calculated as the product of the area of the film coated on the substrate and the thickness measured in surface profilometer. The saturation magnetization is the saturation magnetic moment per unit volume of the film. We have plotted $M_{\mathrm{S}} / M_{\mathrm{S}}$ (bulk), where $M_{\mathrm{S}}$ (bulk) refers to the saturation magnetization for the bulk nickel having a value of $470 \mathrm{emu} / \mathrm{cc}$.

Atomic force microscopy (Seiko, Japan Model SPA 400) was used to characterize the morphology of the films made on different substrates. The images were acquired in the non-contact dynamic force mode and phase imaging is a popular technique coupled to this mode of operation. When the tip is in momentary contact it is affected by the dynamic properties of the material underneath. The detected signal from the cantilever by the photodiode has a slight phase lag from the driving signal sent to the piezo actuator. As a consequence, the variations in microstructure due to the presence of different elements, partial oxidation, granularity as well as evolution of the grain structure can be analyzed much better using these phase contrast images in contrast to the topographical images. We demonstrate that this technique is particularly useful in the case of magnetic thin films where the properties are critically dependent on the surface roughness and the distribution of grain size and the ratio of grain-to-grain boundary area.

\section{Results and discussion}

The XRD patterns of Ni films of thickness ranging from 90-500 nm on BSG substrates are shown in figure 1(a). The $500 \mathrm{~nm}$ nickel film exhibits peaks representing four planes viz. [111], [200], [220], [311] that are observed at thicknesses down to $130 \mathrm{~nm}$. However, for the $90 \mathrm{~nm}$ film only one peak representing the plane [111] is visible, which is no longer observed at $50 \mathrm{~nm}$. The XRD patterns of nickel films of thickness, $50 \mathrm{~nm}$, on different substrates have been shown in figure 1(b). On all the substrates viz. silicon, $\mathrm{MgO}$ and glass substrates, films are amorphous at $50 \mathrm{~nm}$ thickness.

XRD patterns of these films deposited at ambient, 503, 543 and $593 \mathrm{~K}$ substrate temperatures, respectively are shown in figure 2 . The figure clearly shows the evolution of long-range order at relatively higher substrate temperature deposition condition. For example, at the substrate temperature of $503 \mathrm{~K}$, one peak corresponding to [111] appears (figure 2(b)) and at $543 \mathrm{~K}$, the two peaks viz. [111], [200] appear (figure 2(c)) with [200] peak being relatively lower in intensity. The predominantly [111] texture is retained even at $593 \mathrm{~K}$ substrate temperature. The absence of long-range order even at substrate temperature as high as $450^{\circ} \mathrm{C}$ and $50 \mathrm{~nm}$ thick Fe-Ni films has been reported earlier. On crystallization, the films in that study also exhibited a predominant [111] texture (Kim et al 2003).

The microstructural evolution with thickness for nickel thin films coated at ambient temperature is shown in fig- 
ure 3. At $50 \mathrm{~nm}$ thickness, nanoparticles are visible (figure $3(\mathrm{a})$ ) in the microstructure while at $100 \mathrm{~nm}$ thickness clustering of nanoparticles (figure 3(b)) is apparent. At $150 \mathrm{~nm}$ the island-like overlayer on a continuous layer at the bottom can be observed clearly (figure 3(c)).

The role of the substrates in determining the morphological features is shown in figures 4(a), (b) and (c), respectively for nickel thin films coated on glass, $\mathrm{MgO}$ and

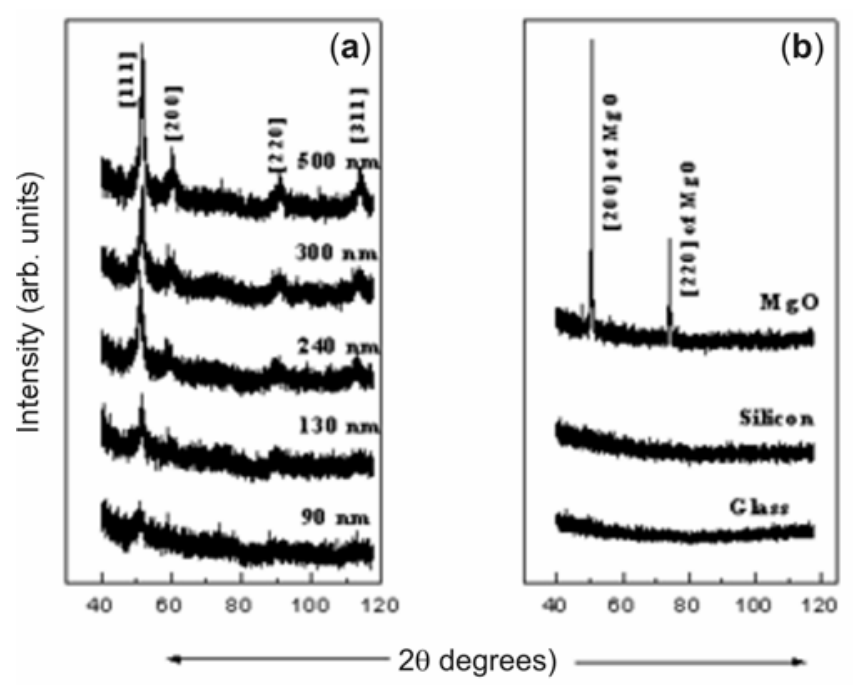

Figure 1. XRD of nickel thin film coated on glass substrate for the thickness of coating (a) different thicknesses and (b) $50 \mathrm{~nm}$ film on different substrates.

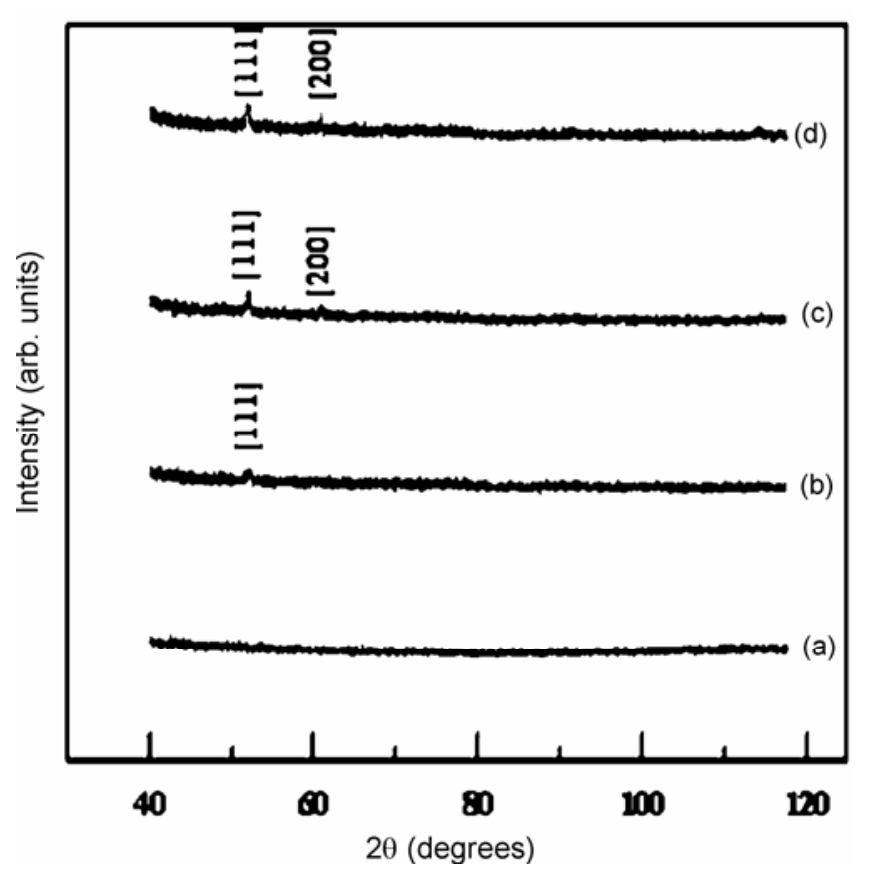

Figure 2. XRD of nickel thin film coated on glass substrate at substrate temperatures (a) $R T$, (b) $503 \mathrm{~K}$, (c) $543 \mathrm{~K}$ and (d) $593 \mathrm{~K}$. silicon, respectively. Figure 4(d) shows the microstructural features for $70 \mathrm{~nm}$ nickel thin film on silicon. Borosilicate glass gives rise to nanoparticulate nickel thin films having a distribution in particle size while $\mathrm{MgO}$ gives rise to columnar kind of growth and silicon substrate interestingly gives rise to self-organization of nanoparticles. Nickel nanoparticles of diameters, 80$120 \mathrm{~nm}$, have self-organized up to $3-4 \mu$ of length on silicon substrate. Further increasing the thickness to $70 \mathrm{~nm}$, silicon substrate gives rise to the growth of linear chains of average diameter 150-200 nm.

The microstructural evolution of the $\mathrm{Ni}$ films coated onto glass substrates at elevated substrate temperatures was investigated by means of dynamic force microscopy both in the topography (figures $5(\mathrm{a})-(\mathrm{d})$ ) and phase contrast (figures 6(a)-(d)) image modes. For ambient temperature (RT) deposited thin films, spherical particles of average diameter of $30-50 \mathrm{~nm}$ are visible (figure 5(a)) with a clear island like microstructure. At $503 \mathrm{~K}$ substrate temperature, spherical particles of diameter, 100-120 nm, form (figure 5(b)). At $543 \mathrm{~K}$ substrate temperature grain size is in the range of 120-150 nm (figure 5(c)) that further increases to result in elongated grains with average diameter of $\sim 150 \mathrm{~nm}$ (figure $5(\mathrm{~d})$ ) at $593 \mathrm{~K}$ substrate temperature. The effect of increasing substrate temperature is therefore to increase the grain size of the films and cause densification resulting in a completely continuous film.

The phase contrast images for these films reveal the microstructural evolution much more explicitly. Figures 6(a) to (d) correspond to the phase contrast images of the films deposited at temperatures from $R T$ to $593 \mathrm{~K}$ and are for the same samples shown in figures $5(a)$ to (d). It is evident that at ambient temperature the island like structure also has discontinuities that are of the order of the size of the grains. As the substrate temperature is increased the increase in grain size is evident, but another interesting feature that is also revealed is that the width of the grain boundary walls decreases as a function of the increase in substrate temperature. The grain boundary walls have been indicated on the images. It thus follows that, as the substrate temperature is increased not only is there an increase in the grain size but there is also an increase in the ratio of the grain to grain boundary area that is the primary reason for densification of the films. At room temperature deposition, the grain boundaries are 20$40 \mathrm{~nm}$ thick with a corresponding grain size of 30-50 nm. At $593 \mathrm{~K}$ substrate temperature while the grain size is $150 \mathrm{~nm}$ or greater, the grain boundaries are between 20 and $50 \mathrm{~nm}$ (figure 6(d)). It was also evident from the phase image and the topographical images that the aspect ratio of the grains increases with increase in substrate temperature indicating larger shape anisotropy.

For nickel films on BSG substrate, there is a grain size distribution, spanning from $30-50 \mathrm{~nm}$. This picture shows the nanoparticulate nature of the thin film at $50 \mathrm{~nm}$ thickness (figure 4(a)). Interestingly, on silicon substrates the 

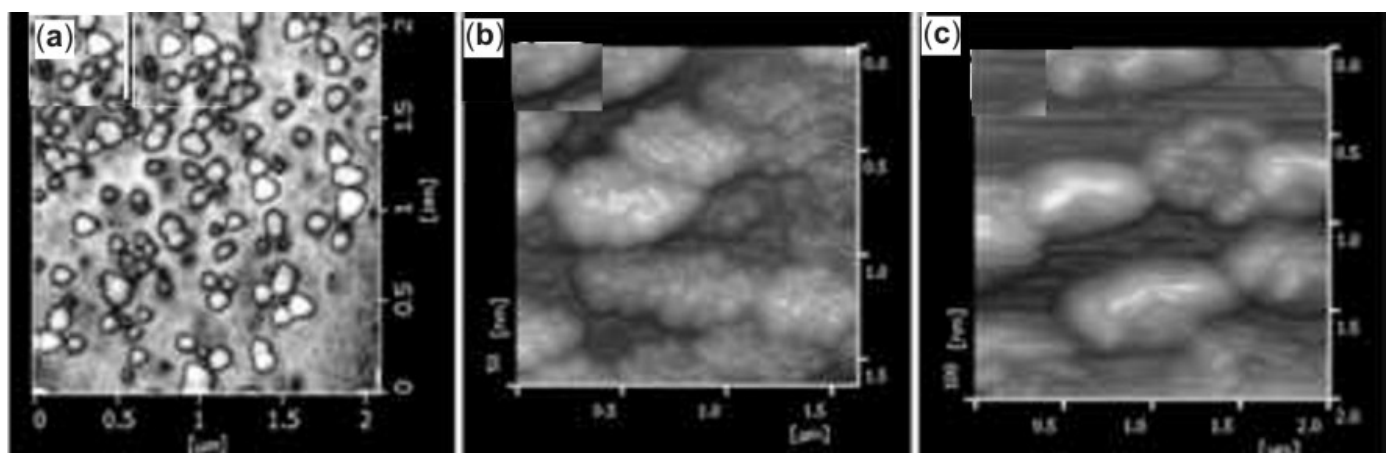

Figure 3. Atomic force microscopic images in DFM mode for thicknesses (a) $50 \mathrm{~nm}$, (b) $100 \mathrm{~nm}$ and (c) $150 \mathrm{~nm}$.
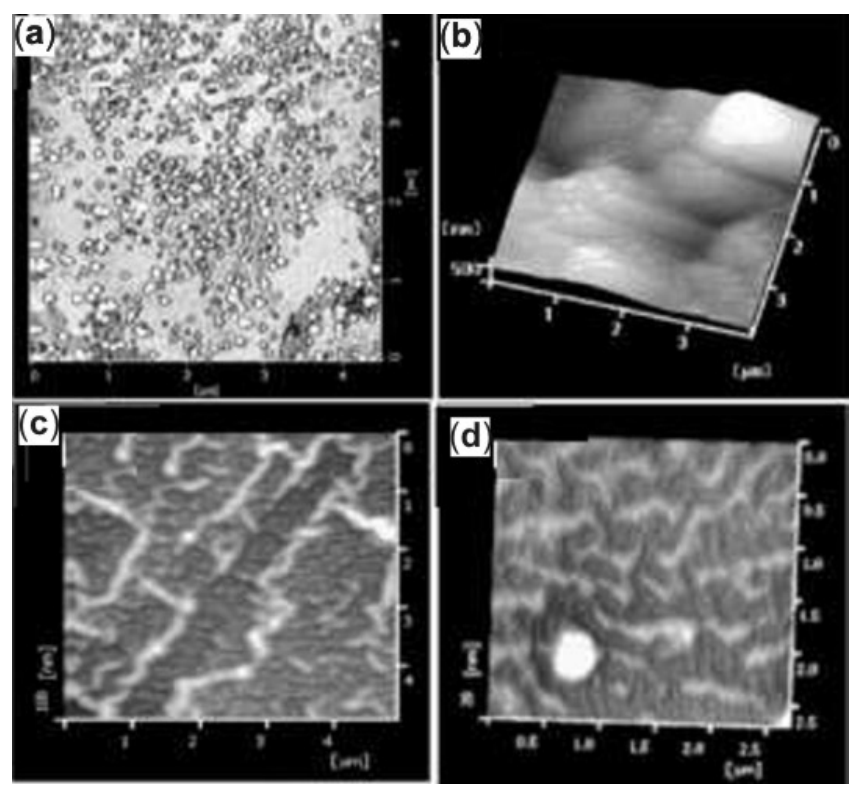

Figure 4. Atomic force microscopic images in DFM mode for nickel thin films (a) for $50 \mathrm{~nm}$ nickel thickness on BSG substrate, (b) $50 \mathrm{~nm}$ thickness on $\mathrm{MgO}$ substrate, (c) $50 \mathrm{~nm}$ thickness on silicon substrate and (d) $70 \mathrm{~nm}$ thickness on silicon substrate.

nanoparticles seem to self-organize in the form of long chains up to 3-4 micron length as shown in figure 4(c). On $\mathrm{MgO}$ substrate the grains are large and island like and show columnar growth feature. It appears that the crystallites aggregate into large grains in all cases. Furthermore the growth seems to follow a growth pattern that involves the formation of a monolayer followed by the formation of $2 \mathrm{D}$ islands that eventually form a dense layer and so on.

The observations of thin film growth at elevated substrate temperature are in qualitative agreement with models proposed for grain growth and preferred texture in thin films as well as their experimentally observed impact on magnetic properties (Bertotti 1998; Barmak et al 2006). For example, it has been proposed (Barmak et al 2006)
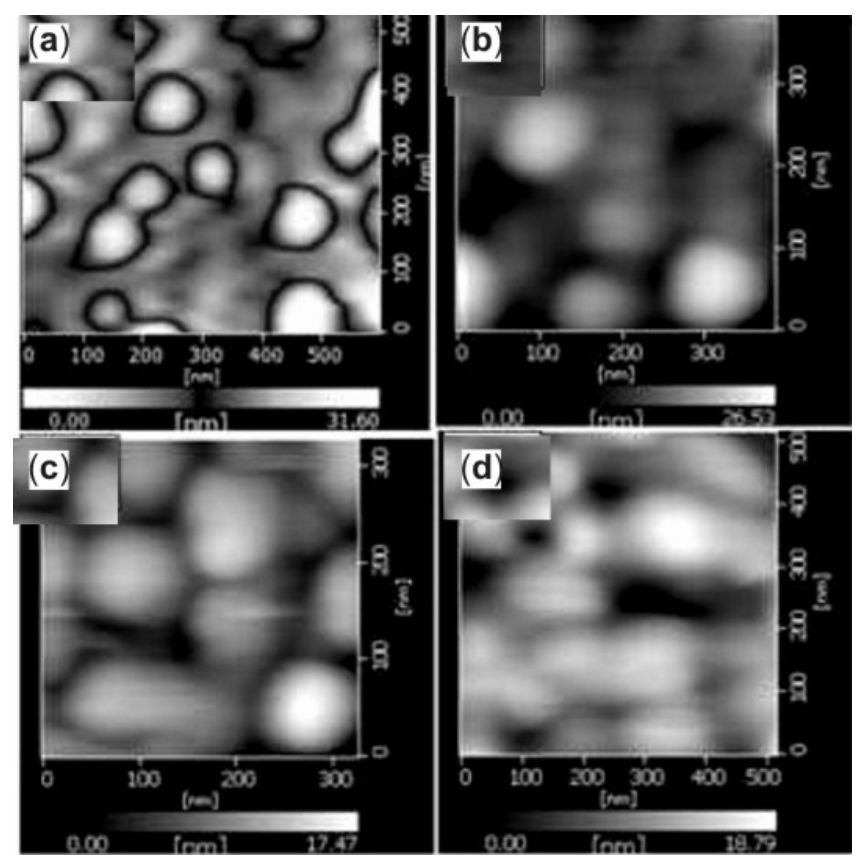

Figure 5. Atomic force microscopic topographical images for $50 \mathrm{~nm}$ thin film coated at different substrate temperatures for substrate temperatures (a) $R T$, (b) 503, (c) 543 and (d) $593 \mathrm{~K}$, respectively

that when the nuclei grow as spherical-cap-shaped islands, they will be surrounded by a zone of width $d$, in which any atom deposited on the substrate surface within a distance $d$, of a pre-existing island will diffuse to that island and become part of it instead of becoming part of a new nucleus. The width, $d$, is a function of substrate temperature and the rate of deposition. The observed microstructure in the present case clearly provides evidence for the existence of this diffusion width in the form of grain boundary walls as shown in figure 6. Other models (Bertotti 1998) predict the stagnation or retardation of grain growth observed in the present case. The stagnation is due to the competition between and minimization of the driving forces for grain growth, which include grain 
boundary, surface and elastic-strain energies. As observed in the present case it has been proposed that the intrinsic stress will be very large in the initial stages of growth and relaxation will be observed as substrate temperature increases for a given thickness. Interestingly, it has been shown that the predominantly [111] texture for $f c c$ metal thin films is possible due to surface energy minimization at high substrate temperatures whereas the [100] texture dominates at lower substrate temperatures. This is also in qualitative agreement with the results of the present study.

According to Gafner et al (2004), formation of a cluster structure depends on the cooling conditions usually after the onset of crystallization. It was demonstrated that for slow cooling condition, $f c c$ lattice of nickel evolves and for the rapid cooling condition icosahedral structure evolves. In the current work, cluster of nanoparticles have been observed for ambient temperature deposition conditions indicating quenching of the vapour molecules on impact with the substrate surfaces. As a consequence, when an island forms, strain will be larger at the rim of the particles due to surface tension effect because of quenching. Therefore, the next particle landing on the substrate will preferentially occupy the rim position and so on, resulting eventually in self-organized growth. At higher substrate temperature, however, where large kinetic energy is available for the dynamic movement of the particles and for relaxation to occur, strain would be minimized and self-organization would be less probable.
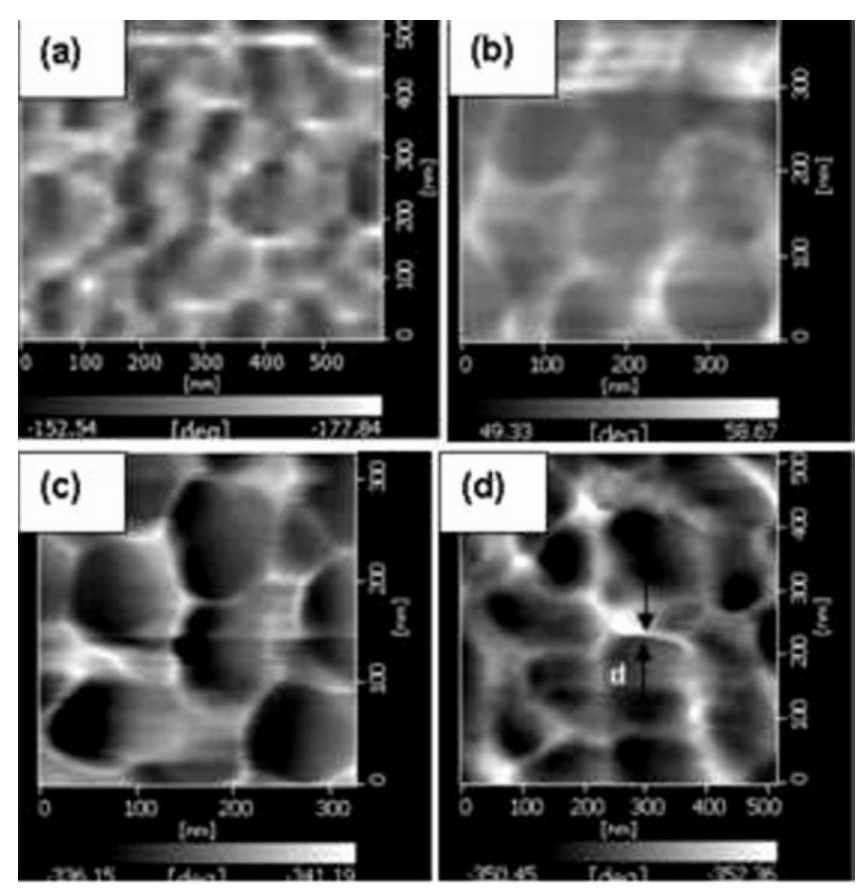

Figure 6. Atomic force microscopic phase contrast images for $50 \mathrm{~nm}$ thin film coated at different substrate temperatures for substrate temperatures (a) $R T$, (b) 503, (c) 543 and (d) $593 \mathrm{~K}$, respectively (d is the grain boundary wall width).
Fujikawa et al (2006) proposed that the interaction of nickel clusters and the support surface regulates the nickel thin film feature size. Hence, as observed here, the selection of the support surface plays a dominant role in determination of the organization shape.

The VSM hysteresis loops for nickel for different thicknesses are shown in figure 7(a). It is observed that the coercive field reduces to zero, as the thickness of nickel film is decreased. Figure 7(b) represents hysteresis loops of $50 \mathrm{~nm}$ nickel films on different substrates. The coercive field for the films on the glass is lower than those for the films on silicon and $\mathrm{MgO}$ substrates. This may be due to the better short-range order of the films deposited on the crystalline substrates. From the VSM hysteresis loops (figure 8) recorded for films deposited at various substrate temperatures it can be observed that coercive field first increases with increase in substrate temperature from $R T$ to $503 \mathrm{~K}$ (figures $8(\mathrm{a})-(\mathrm{c})$ ) and then decreases with the rise of substrate temperature up to $593 \mathrm{~K}$ (figure $8(\mathrm{~d})$ ). The normalized saturation magneti-

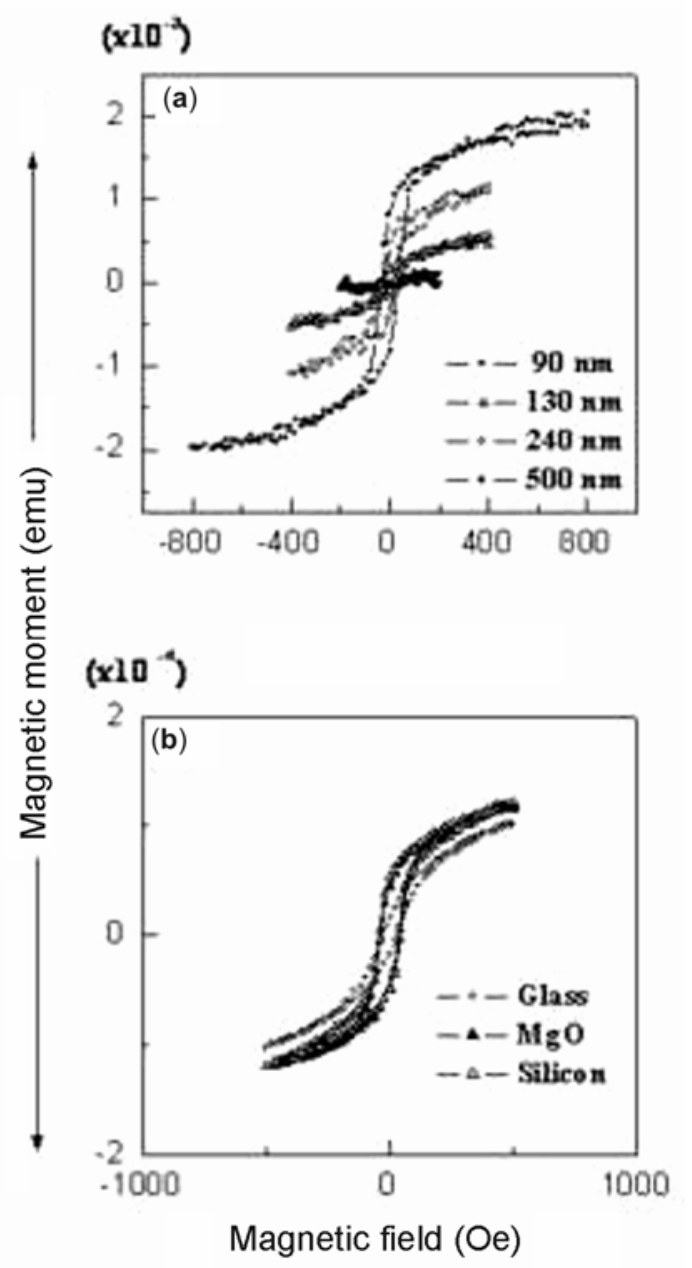

Figure 7. Vibrating sample magnetometric hysteresis loops for nickel thin films for (a) different thicknesses and (b) different substrates. 

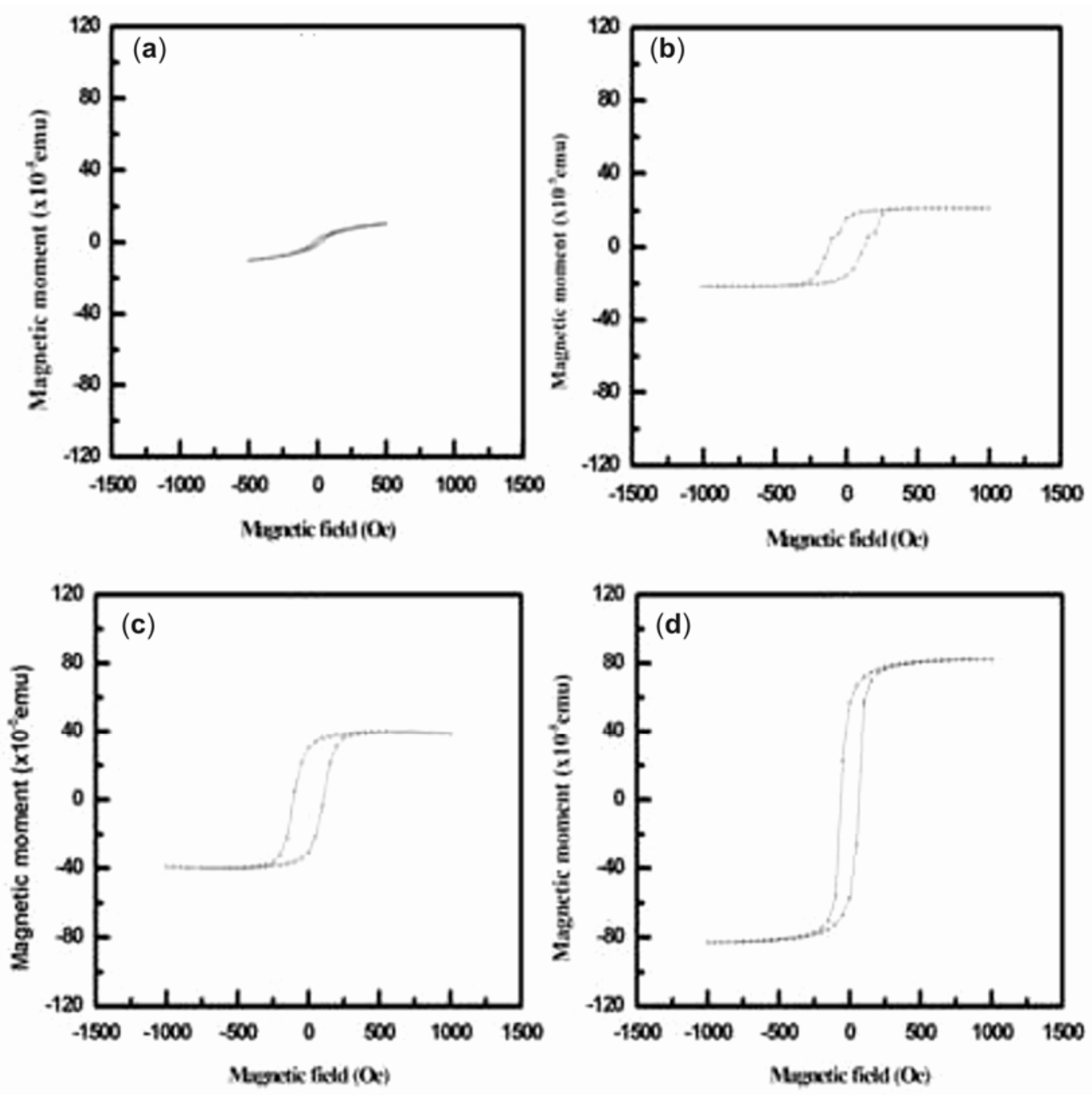

Figure 8. VSM hysteresis loops for nickel thin film on glass substrate of same thickness, $50 \mathrm{~nm}$, at different substrate temperatures (a) $R T$, (b) 503, (c) 543 and (d) $593 \mathrm{~K}$.

zation is enhanced and saturation field is reduced at elevated substrate temperature. Clearly at $R T$ and up to $503 \mathrm{~K}$ substrate temperature, the thin film is completely in the nanocrystalline region, but on further increase in substrate temperature it makes a transition to the crystalline region (long range order evolves), causing a decrease in the coercive field beyond $503 \mathrm{~K}$ substrate temperature, symbolizing crystalline magnetic behaviour.

The variation of coercive field, crystallite size and magnetization energy density for nickel films (of thickness range 500-90 nm) with thickness is shown in figures 9(a), (b) and (c), respectively. Crystallite size is found in the range $5-9 \mathrm{~nm}$. The crystallite size gradually increases with the increase of thickness of the film. However, both coercive field and magnetization energy density first increase and then at large thickness start reducing.

The trends of crystallite size, grain size, and surface roughness, coercive field and normalized saturation magnetization as a function of the substrate temperature is shown in figure 10. Crystallite size increases with the increase of substrate temperature while coercive field too increases up to $503 \mathrm{~K}$ and then decreases. Saturation magnetization increases with the substrate temperature very slowly up to $503 \mathrm{~K}$ and rapidly thereafter. Surface roughness (RMS) increases from $0.73 \mathrm{~nm}$ to $3.42 \mathrm{~nm}$ while substrate temperature rises from $R T$ to $593 \mathrm{~K}$. This result is in agreement with the report (Ha et al 1999) on microstructural growth of $\mathrm{Cu}$ thin films with the substrate temperature. Residual strain increases with the decrease in thickness (figure 11). Similar large strain has been reported earlier (Thompson 1999) at this thickness range for $R T$ deposition conditions. As the substrate temperature increases to $593 \mathrm{~K}$, the residual strain is negligibly small indicating that the system is more relaxed at higher substrate temperature.

Finite size effects have been examined earlier theoretically (Kachkachi et al 2000) and experimentally (Ohandley 2000; Battle and Labarta 2002; Zhang et al 
2004). Coercive field for thin film varies as $H_{\mathrm{ci}} / H_{\mathrm{ci} 0}=$ $1-\left[D_{\mathrm{p}} / D\right]^{3 / 2}$ when its thickness is cut (Cullity 1972), where $H_{\mathrm{ci} 0}$ is $2 K_{\mathrm{u}} V / \mu$ (when $T=0$ ), $K_{\mathrm{u}}$ the uniaxial anisotropy constant, $V$ the volume of the ferromagnet, and $D$ the diameter of the magnetic particles. Below a critical diameter, $D_{\mathrm{p}}$, coercivity is zero, because of thermal effects that are strong enough to spontaneously demagnetize the previously saturated assembly of particles. However, geometry, finite size effects and structure can all have significant effect on the magnetization response of nanoparticles. Coercive field trend with cluster size of the films as modeled atomistically by Zheng (Zheng et al 2003) using Monte Carlo Simulation supports our results.

The crystalline to nanocrystalline transition in magnetic behaviour due to size effect has been illustrated and explained earlier (Herger 1992; Chan et al 2006). Nanocrystalline magnetic behaviour is expected in island metallic films (IMF) while crystalline magnetic behaviour is expected in continuous metallic films (CMF). There is, however, no sharp division of these two regimes viz. IMFs and CMFs. Similarly, the transition from crystalli-
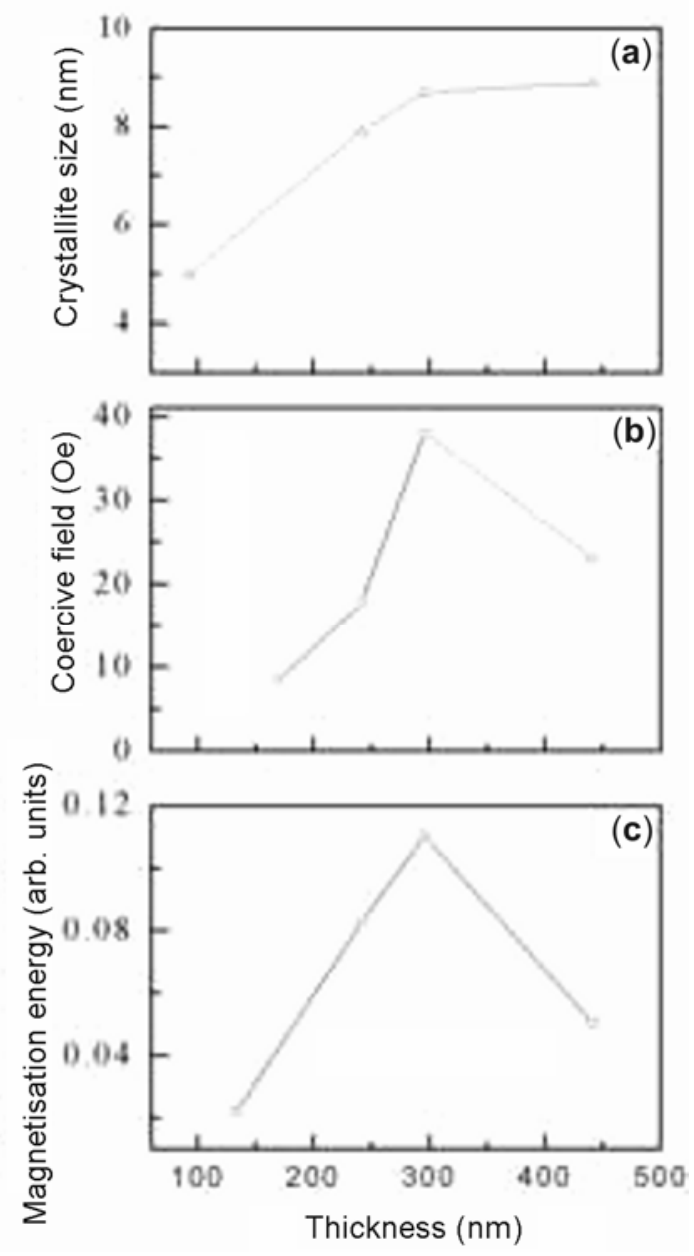

Figure 9. Trend with the thickness of nickel thin films for (a) crystallite size, (b) coercive field and (c) magnetization energy density. nity to nanocrystallinity is exhibited over a window of thickness where there is large variation in crystallite size and some volume of the material is amorphous. In the present study, long-range order is established only for substrate temperatures, $\geq 503 \mathrm{~K}$, as evidenced from the $\mathrm{X}$-ray diffraction patterns. Grain size too increases as the substrate temperature is increased to $503 \mathrm{~K}$. From 503$593 \mathrm{~K}$ substrate temperature, grain size as well as crystallite size both increase and residual strain reduces, and as a consequence coercive field is reduced. The decrease in coercive field may also be due to two other reasons; first low thickness $(50 \mathrm{~nm})$ and second, island growth is

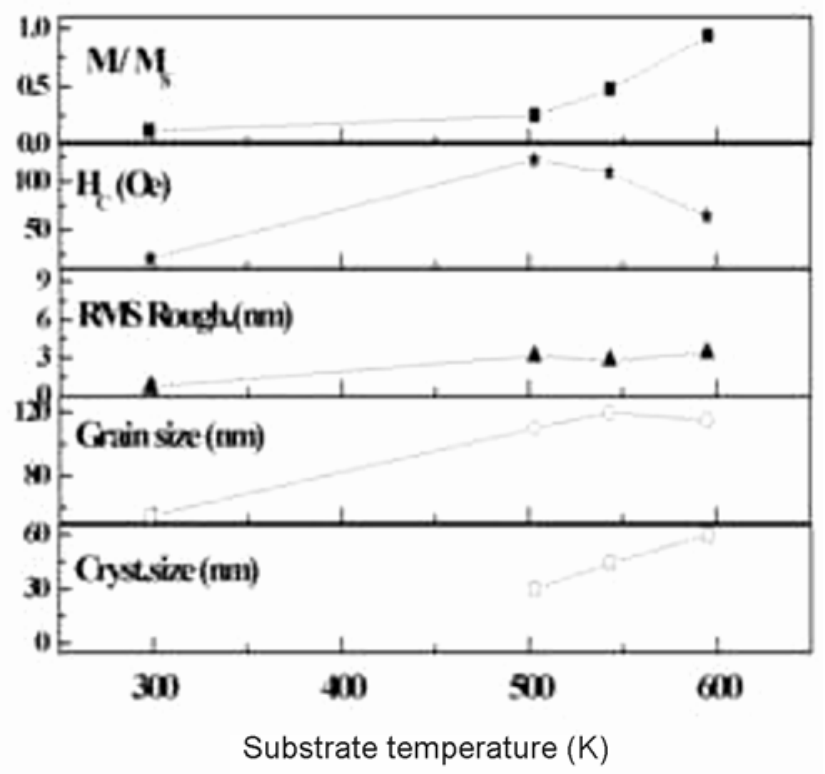

Figure 10. Variation of crystallite size (nm), grain size $(\mathrm{nm})$, RMS roughness, coercive field and normalized saturation magnetization vs substrate temperature for $50 \mathrm{~nm}$ thin films.

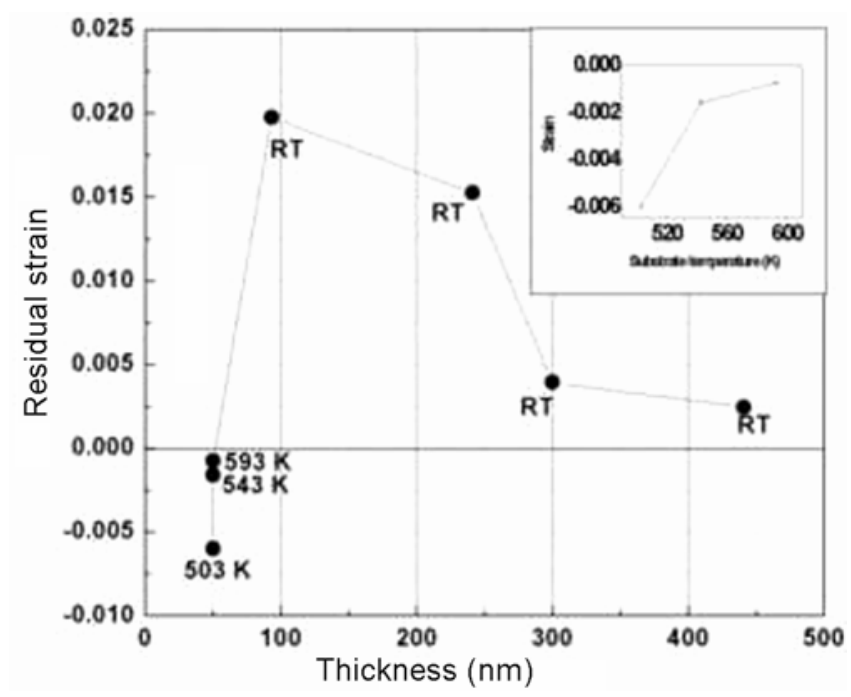

Figure 11. Residual strain vs thickness vs substrate temperature for $50 \mathrm{~nm}$ thin film, inset is residual strain vs substrate temperature. 
favoured at lower deposition temperature in thermally evaporated films. There will, therefore, be a large microstructural change during the transition from island to continuous thin film with the rise in substrate temperature giving rise to such a change in magnetic behaviour. Simulations (Savaloni and Shaharaki 2004) based on structural zone model for growth at different substrate temperatures also are in agreement with our results.

\section{Conclusions}

The effect of thickness and substrate temperature on the microstructure, structure and magnetic properties of $\mathrm{Ni}$ thin films has thus been demonstrated. It is shown that grain area as well as the grain boundary width stagnates beyond a certain value, with increase in substrate temperature. Phase contrast microscopy has been used to illustrate this effect. The magnetic properties such as coercive field and saturation magnetization are shown to be critically dependent on the thickness as well as substrate temperature. Thin film growth depends heavily on the thickness of thin films. Substrates have been found to affect the microstructural growth features of thin films as well as the subsequent magnetic behaviour. Some interesting features like self-organization of nickel nanoparticles has been observed.

\section{Acknowledgements}

The authors acknowledge the funding from the University Grants Commission for Centre of Advanced Studies in Physics, especially a fellowship for (PK). Facilities from UGC under UPE and SAP programs, and DST-FIST program are also acknowledged.

\section{References}

Amoruso S, Ausanio G, Lisio C D, Iannotti V, Vitiello M, Wang X and Lanotte L 2005 Appl. Surf. Sci. 24771

Aurongzeb D, Ram K B and Menon L 2005 Appl. Phys. Lett. 87 172509

Barmak K et al 2006 Scr. Mater. 541059

Battle X and Labarta A 2002 J. Phys. D: Appl. Phys. 35 R15

Bertotti G 1998 Hysteresis in magnetism (London, UK: Academic Press) p. 375

Castaldi L, Gibbs M R J and Davies H A 2004 J. Appl. Phys. 96 5063

Chan K Y, Tou T Y and Teo B S 2006 Microelectronics J. 37930

Chang S W, Liu J H and Lue J T 2003 Meas. Sci. Technol. 14583

Cullity B D 1972 Introduction to magnetic materials (MA: Addison-Wesley) p. 387

Dirks A G and Leamy H J 1977 Thin Solid Films 47219

Flynn D and Desmulliez M 2006 J. Phys.: Conf. Ser. 34112

Fujikawa K, Suzuki S, Koike Y, Chun W J and Asakura K 2006 Surf. Sci. 600 L 117
Gafner Y Y, Gafner S L and Entel P 2004 Phys. Solid State 461327

Girgis E, Pogossian S P and Benkhedar M L 2006 J. Nanosci. Nanotechnol. 61135

Guan S and Nelson B J 2005 J. Magn. Magn. Mater. 29249

Gupta N, Verma A, Kashyap S C and Dube D C 2007 J. Magn. Magn. Mater. 308137

Ha K, Ciria M, O'Handley R C, Stephens P W and Pagola S 1999 Phys. Rev. B19 13780

Ha N D, Phan M H and Kim C O 2006 J. Appl. Phys. 99 08F 105-1

Haque S A, Matsuo A, Seino Y, Yamamoto Y, Yamada S and Hori H 2001 Physica B305 121

Herger G 1992 J. Magn. Magn. Mater. 112258

Hong J, Gary S W, Jones J W and Moody N R 1997 J. Appl. Phys. 816754

Hsieh C T, Liu J Q and Lue J T 2005 Appl. Surf. Sci. 2521899

Kachkachi H, Nogues M, Tronc E and Garanin D A $2000 \mathrm{~J}$. Magn. Magn. Mater. 221158

Kim J G, Han K H, Song S H and Reilly A 2003 Thin Solid Films $\mathbf{4 4 0} 54$

Klapetek P, Ohlídal I and Buršík J 2007 Meas. Sci. Technol. 18 528

Lukaszew R A, Zhang Z, Stoica V and Clarke R 2003 Appl. Surf. Sci. 21974

Messier R, Giri A P and Roy R A 1984 J. Vac. Sci. Technol. 2 500

Novikov V Y 1999 Acta Mater. 474507

Ohandley R C 2000 Modern magnetic materials (New York: Wiley Interscience) p. 306

Ravinder D, Kumar K V and Reddy A V R 2003 Mater. Letts $\mathbf{5 7} 4162$

Savaloni H and Shaharaki M G 2004 Nanotechnology 15311

Sedlácková K, Lobotka P, Vávra I and Radnóczi G 2005 Carbon $\mathbf{4 3} 2192$

Seel S C, Thompson C V, Hearne S J and Floro J A $2000 \mathrm{~J}$. Appl. Phys. 887079

Serrona L K E B et al 2003 Appl. Phys. Lett. 821751

Shalyguina E E, Mukasheva M A, Abrosimova N M, Kozlovskii L, Tamanis E and Shalygin A N 2006 J. Magn. Magn. Mater. 300 e367

Shimizu H, Hayashi T, Nishinaga T and Tanaka M 1999 Appl. Phys. Lett. $\mathbf{7 4} 398$

Snyder D W, Mahajan S, Ko E I and Sides P J 1991 Appl. Phys. Lett. $\mathbf{5 8} 848$

Swerts J, Temst K, Vandamme N, Haesendonck C V and Bruynseraede Y 2002 J. Magn. Magn. Mater. 240380

Thompson C V 1999 J. Mater. Res. 143164

Wang Y H, Sood D K and Ghantasala M K 2002 Proc. SPIE 4936394

Yang J, Huang Y and Xu K 2007 Surf. Coat. Technol. 201 5574

Yi J B et al 2004 J. Magn. Magn. Mater. 284303

Zhang K, Rotter F, Uhrmacher M, Ronning C, Krauser J and Hofsäss H 2007 New J. Phys. 929

Zhang W H, Sun C Q and Li S 2004 Solid State Commun. 130 603

Zhao Y P, Gamache R M, Wang G C, Lu T M, Palasantzas G and Hosson J T M D 2001 J. Appl. Phys. 891325

Zheng G P, Gross D and Li M 2003 J. Appl. Phys. 937652 